\title{
Model-Driven Derivation of Domain Functional Requirements from Use Cases
}

\author{
Jianmei Guo ${ }^{1}$, Zheying Zhang ${ }^{2}$, Yinglin Wang ${ }^{1}$ \\ ${ }^{1}$ Department of Computer Science and Engineering, Shanghai Jiao Tong University, Shanghai, China; ${ }^{2}$ Department of Computer \\ Sciences, University of Tampere, Kanslerinrinne, Finland. \\ Email: \{guojianmei, ylwang\}@sjtu.edu.cn, Zheying.Zhang@cs.uta.fi
}

\begin{abstract}
Domain analysis is essential to core assets development in software product line engineering. Most existing approaches, however, depend on domain experts' experience to analyze the commonality and variability of systems in a domain, which remains a manual and intensive process. This paper addresses the issue by proposing a model-driven approach to automating the domain requirements derivation process. The paper focuses on the match between the use cases of existing individual products and the domain functional requirements of a product line. By introducing a set of linguistic description dimensions to differentiate the sub-variations in a use case, the use case template is extended to model variability. To this end, a transformation process is formulated to sustain and deduce the information in use cases, and to match it to domain functional requirements. This paper also presents a prototype which implements the derivation as a model transformation described in a graphical model transformation language MOLA. This approach complements existing domain analysis techniques with less manual operation cost and more efficiency by automating the domain functional requirements development.
\end{abstract}

Keywords: Software Product Lines, Domain Analysis, Model Transformation, Use Cases, Functional Requirements

\section{Introduction}

Software product line engineering (SPLE) has emerged as one of the most promising software development paradigms for production of a set of closely related products. It reduces development costs, shortens time to market, improves product quality, and helps to achieve greater market agility [1,2]. Some organizations make a transition from conventional single-system engineering to SPLE in order to enable mass customization and maintain their market presence. They systematically reuse legacy systems and existing products which embody their domain expertise to develop the core assets base of product lines [3,4].

Domain analysis is a process by which information used in developing software systems is identified, captured, and organized with the purpose of making it reusable when creating new systems [5]. Its essential activeties include analysis of commonalities and variabilities from the similar existing products and elicitation of domain requirements. Many domain analysis techniques [6-10] have been used to identify and document the commonalities and variabilities of systems constituting the product line. These techniques, however, mostly de- pend on domain experts' experience [2,11,12], and lack automated support $[2,4,11]$. When existing products have a significant amount of commonalities and also consistent differences among them, it is possible to extract the product line from them. If domain requirements, together with other systems artifacts, can be automatically reasoned and extracted from requirements of existing products, the amount of effort will be reduced significantly.

In this paper, we focus on functional requirements, and propose a model-driven approach to deriving domain functional requirements (DFRs) from use cases of existing products. Use cases are a widely used technique for requirements specification, but there is no generally accepted formalism to explicitly represent the variations of scenarios in a use case [13]. Using the metamodeling [14] and the model transformation techniques [15,16], the use case template [17] is adapted for variability modeling in order to derive DFRs for product lines. By introducing a set of linguistic description dimensions into use cases to differentiate the sub-variations, the use case template is extended to model the variability. Further, we analyze the correlation between the tailored use cases and the DFRs, and present a model-driven framework to derive DFRs from use cases. We also presents a prototype which 
implements the derivation as a model transformation described in a graphical model transformation language MOLA [18]. Our approach reduces the manual operation cost and complements existing domain analysis techniques by introducing an automated process of commonality and variability analysis.

The remainder of this paper is organized as follows. Section 2 defines the metamodels of use cases and DFRs and analyzes their correlation. Section 3 proposes a model-driven framework for DFRs derivation and presents a model transformation definition in MOLA. Section 4 discusses related work. Section 5 concludes this paper with future work.

\section{The Underlying Metamodels}

\subsection{Use Cases}

Use cases have been a means to understand, specify, and analyze user requirements that is rather often used [19]. They are widely adopted to capture functional requirements from an outside or users point of view. A use case describes the actions of an actor when following a certain task while interacting with the system to be described. It also describes a system's behavior as it responds to a request that originates from outside of the system. Use cases are often recorded by following a template. Although there are no standard use case templates, most of them describe more or less the same issues, e.g., the system to be described, the use cases within the system, the actors outside the system, and the relationships between actors and use cases or in between use cases [20].

One of the most commonly used use case templates is suggested by Cockburn [21]. According to Cockburn's template, a use case is described with its name, goal in context, scope, level, trigger, pre- and postconditions, main success scenario, extensions, sub-variations, and other characteristics [17]. The main success scenario describes what the use case actually does. It is the main part in a use case description, and often described as a sequence of steps or several alternatives to steps, such as extensions and sub-variations. Extensions specify changes in the course of execution of the main success scenario, and sub-variations give the further details of a step's manner or mode that will cause eventually bifurcation in the scenario. The main success scenario, together with extensions and sub-variations, describes a use case behavior, and also implies a set of fine-grained functional requirements. As is shown in Figure 1, the step is a basic object to capture a use case behavior. It has attributes such as step \#, name, description, use case name, actor, and trigger. The attributes step \# and name can be used to identify a step. A step could have sub-variations, and can be extended to another (set of) step(s) based on a given condition. Instances of step form the main success scenario.

To derive DFRs from use cases, common and variable requirements have to be identified and analyzes. The above use case description includes the specifications of sub-variations and extensions, but still lacks the formalism to model variability and to support domain analysis. In order to add the formalism to support variability modeling for the product line, we extend the existing use case metamodel by adding the attribute ProductSite and a set of dimensions for the steps described in the main success scenario, as shown in Figure 1. The attribute "ProductSite" records the owner of the step, which clarifies the existing product to which the step belongs, and help to analyze the commonality and variability in the course of defining the product line scope. The dimensions structure the specification of the sub-variations for the steps. They, are deduced according to the linguistic characteristics of functional requirements [4] and present the different perspectives of sub-variations. The dimensions include agentive, attributive, locational, temporal, process, and purpose.

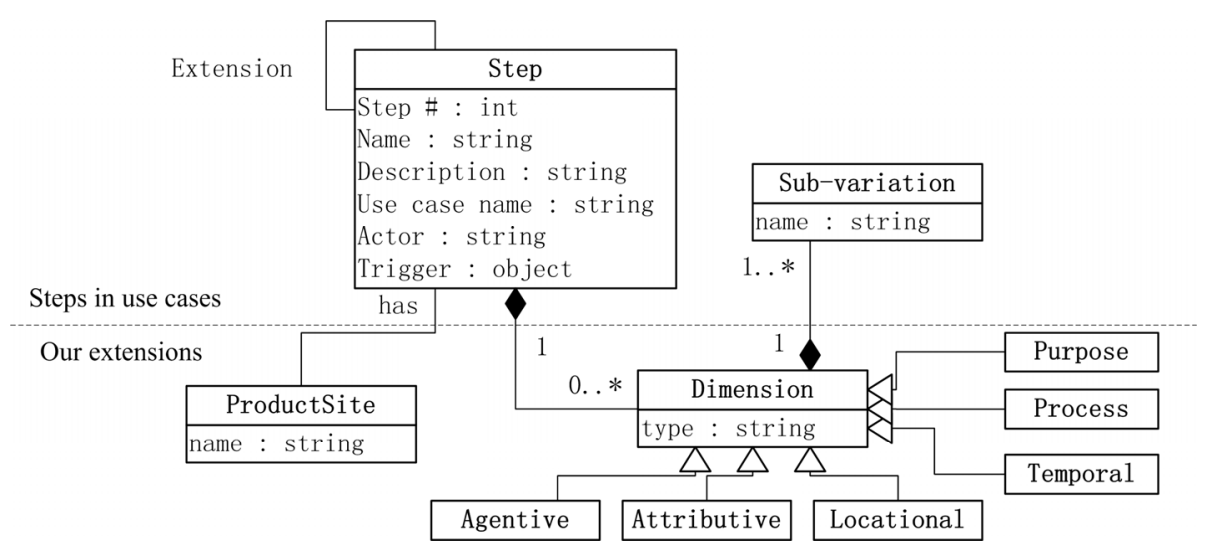

Figure 1. The extended use case metamodel. 
Agentive defines the agent(s) whose activities will bring about the state of affairs implied by the step, e.g., "\{author $\}_{\text {Agentive }}$ submits an article". Attributive defines the attributes of the agentive or of the object associated with the action implied by the step, e.g., "submit a \{research, review $\}_{\text {Attributive }}$ article". Locational defines the spatial location(s) where the activity implied by the step is supposed to take place, e.g., "submit an article \{at office, at home $\}_{\text {Locational". Temporal defines the duration or }}$ frequency of the activity implied by the step, e.g., "submit an article \{every week, every month $\}_{\text {Temporal". Proc- }}$ ess defines the instrument, the means and the manner by which the activity is performed, e.g., "submit an article by $\{\text { email, submission system }\}_{\text {Process". Purpose defines }}$ the purpose that the agentive carries out the activity or that the objective is affected by the activity implied by the step, e.g., "submit an article for \{propagating the knowledge $\}_{\text {Purpose" }}$.

The extended use case metamodel mainly involves the steps, the sub-variations and the extensions because these elements from Cockburn's template have the direct relationship with functional requirements. It also models the variability through introducing a set of linguistic description dimensions to differentiate the sub-variations. The product site of each step is added for further analyzing the commonality and variability of DFRs.

\subsection{Domain Functional Requirements}

DFRs specify the common and variable requirements for all foreseeable applications of the product line. According to the Orthogonal Variability Model (OVM) [2], we define the variability model of DFRs in Figure 2.

Besides the essential attributes of a DFR, i.e. name and description, a DFR shall document its variability. Variability comprises the variability subject and the variability object. A variability subject is a variable item or a variable property of such an item [2]. If a DFR or its property has the tendency to change, it is a variability subject. The property with tendency to change can be further defined as a variation point. A DFR could have one or more variation points. In general, a variation point of a DFR expresses a variable semantic concern of the DFR. To present different semantic concerns of a DFR, we also define a set of types for the variation points of DFRs according to the linguistic characteristics of functional requirements [4], i.e. agentive, attributive, locational, temporal, process, and purpose.

A variability object is a particular instance of a variability subject [2]. It is represented as a variant. A variant represents a single option of the semantic concern that the variation point expresses, and a variation point may have one or more variants.

A DFR could come from one or more product sites in a product line. Such product sites can be used to calculate the $C V$ ratio (Commonality/Variability ratio). A CV ratio records the frequency a DFR appears in a domain. Engineers can use the CV ratios to decide whether a DFR is common or optional. Generally, a DFR is common if it has a CV ratio " $100 \%$ ".

A DFR constraint documents a relationship between two DFRs, between a variant and a DFR, or between two variants. There are two types of relationship, i.e. requires and excludes. Each DFR constraint has a domain and a range. A domain of a constraint is the constraint's subject that possesses the constraint relation; a range of a constraint is the constraint's object that is affected by the constraint relation. For example, a constraint "A requires $\mathrm{B}$ " expresses the constraint relation "requires" between its domain "A" and its range "B". A domain or a range also has its "name" and its "type".

\subsection{Correlation between Use Cases and Domain Functional Requirements}

Steps presented in our prior metamodel describe the

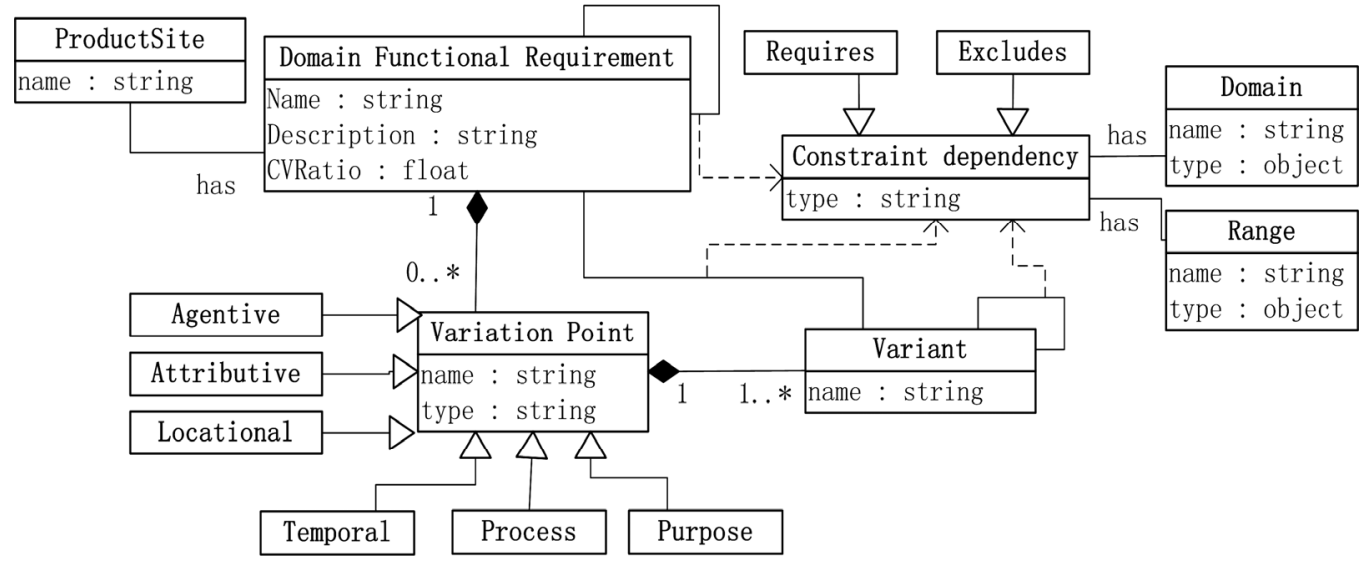

Figure 2. The domain functional requirement metamodel. 
fine-gained functional requirements of a use case. The same requirements can be specified as a DFR for a product line. Since different products could have the same, the similar, or the different functional requirements, the DFRs can be obtained by merging the same and the similar functional requirements and distinguishing the variable ones. We conclude the correlation between use cases and DFRs as follows, which grounds the model transformation for DFR derivation.

\subsubsection{Identifying Requirements}

Steps with different ProductSite value is mapped into a DFR if their identity has the same value, i.e., the same attribute name. Then, the sub-variations of a step are mapped into the variants of the DFR according to their same names. The dimensions of the sub-variations are also mapped into the variation points of the DFR according to their same types.

\subsubsection{Analyzing Commonality and Variability}

The commonality and variability can be analyzed by calculating the CV ratio. The productSite of the same step, together with the total number of products of the product line, forms the input of the calculation.

\subsubsection{Identifying the Constraints}

The relationships between and within the use cases shall be mapped correctly into the constraints of DFRs in terms of the same domain and the same range. Some constraints are straightforward and can be identified easily. For example, the extensions and the triggers of the steps can be mapped into the "requires" constraints of DFRs, i.e. "a trigger of a step" can be represented as "the step requires the trigger". The "precondition" of a use case can be mapped into the "requires" constrains of the DRF derived from the step numbered Step 1 in the use case. However, some more complex relationships in use cases are not identified in this paper. For example, according to Cockburn's template, a step also represents another use case (subordinate use case). Thus, a complete use case could have different "levels". These levels form the hierarchical relationship between the steps. In addition, the conditional relationship and the sequential relationship of the steps are also simplified. These complex relationships will be explored in our future work.

\section{The Derivation Process}

\subsection{Framework}

Based on the proposed metamodels and their correlation, it is feasible to derive the DFRs from the use cases of a set of closely related products in the same domain. We propose a model-driven framework for DFRs derivation from use cases in Figure 3.

The figure shows the scenario of model transformation from multiple source models (use cases) into a target model (DFR model). Both the source model and the target model are instantiated from their respective metamodels, i.e., the use case metamodel and the DFR metamodel. A model transformation definition is defined based on these metamodel specifications. The transformation definition is executed on concrete models by a transformation engine.

\subsection{Model Transformation Process}

We present a prototype that implements the derivation of DFRs as a model transformation described in a graphical model transformation language MOLA [18]. MOLA provides an easy readable graphical transformation language by combining traditional structured programming in a graphical form with pattern-based rules. It clearly distinguishes what is from source models, what is from target models, and what is the mapping association between them. It is suitable for representing loops, which makes the time complexity analysis clearer.

A program in MOLA is a sequence of statements. A statement is a graphical area delimited by a rounded rectangle. The statement sequence is shown by dashed arrows. Thus, a MOLA program actually is a sort of a "structured flowchart". The simplest kind of statement is a rule that performs an elementary transformation of instances. A rule contains a pattern that is a set of elements representing class and association instances (links) and is built in accordance with the source metamodel. A rule has also the action that specifies new class instances to be built, instances to be deleted, association instances to be built or deleted, and the modified attribute values. The

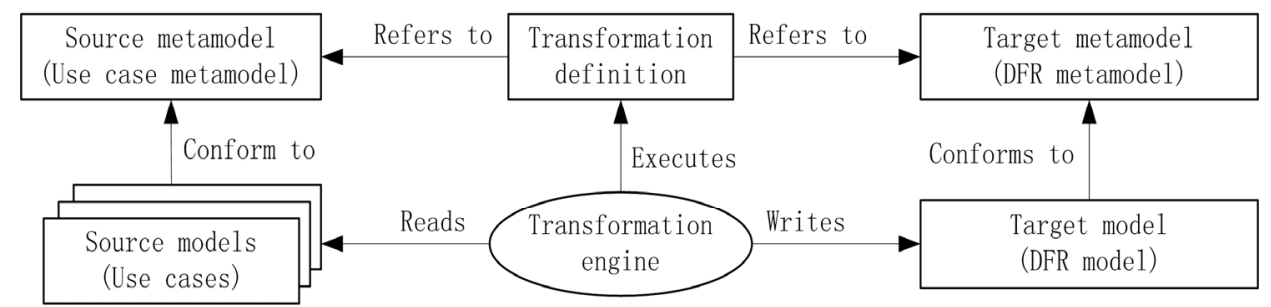

Figure 3. Model-driven framework for DFRs derivation. 
semantics of a rule is standard, i.e., locating a pattern instance in the source model and apply the actions.

The new elements, instances, and links, are shown with dotted lines. The mapping associations prefix the association name by the "\#” character. The mapping associations link instances corresponding to different metamodels; they typically set the context for next subordinate transformations and trace instances between source models and target models in the model transformation.

The most important statement type in MOLA is the loop. Graphically a loop is a rectangular frame, containing a sequence of statements. This sequence starts with a special loop head statement. The loop head is also a patter but with the loop variable highlighted (by a bold frame). The reference notation prefixes an instance name by the “@” character to show that the same instance selected by the loop head is used. The semantics of a loop is natural, i.e., performing the loop for any loop variable instance which satisfies the conditions specified by the pattern.

As is shown in Figure 4, the model transformation

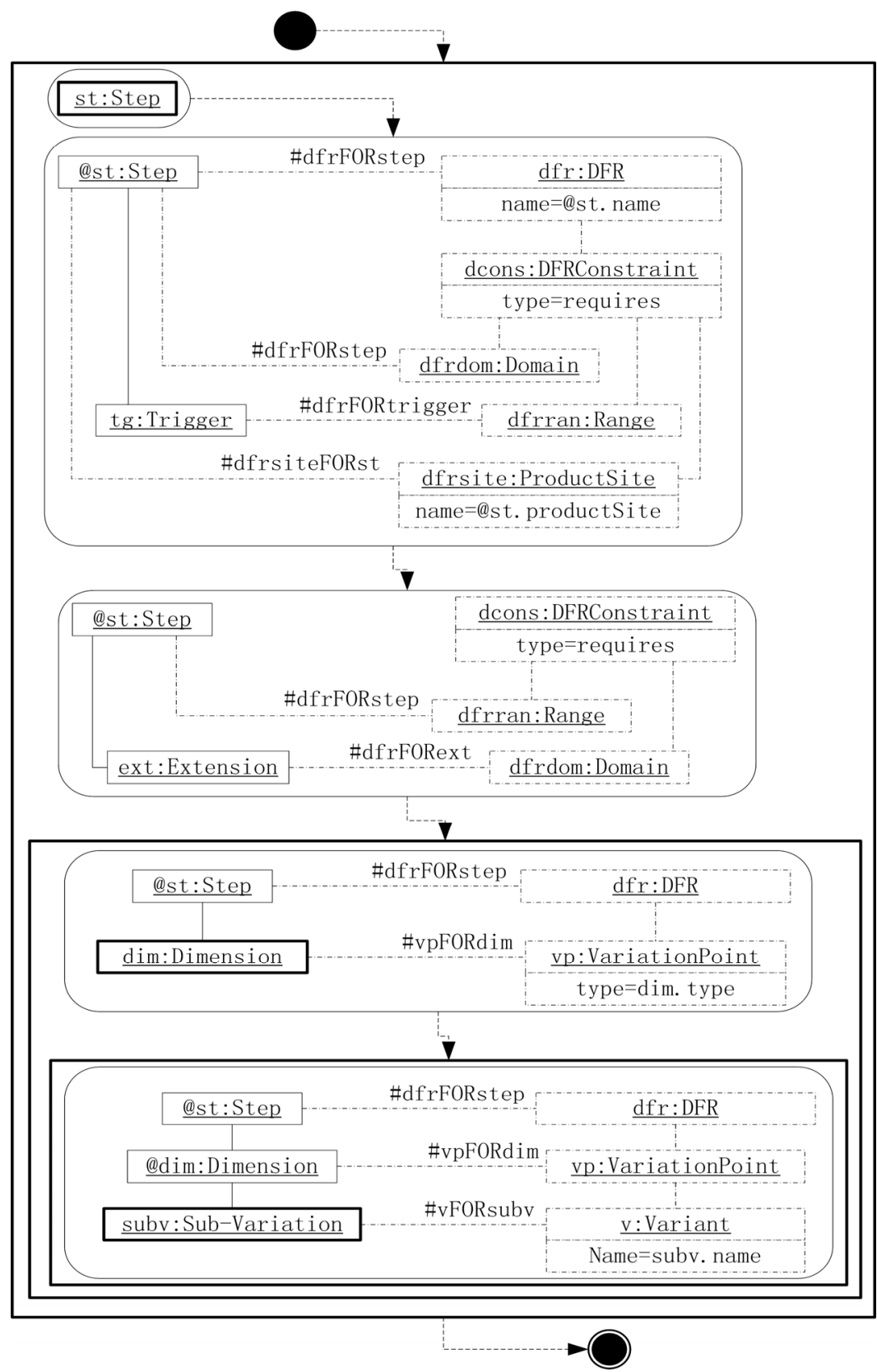

Figure 4. Model transformation definition in MOLA. 
from use cases to DFRs contains three nested loops. The outer loop is executed for each step instance. The next statement is a rule building a DFR from a set of steps according to their same names. The mapping association "\#dfrFORst" records which DFR from which step actually has been generated and can be reused in the following statements. Thus, all input steps with the same name will be merged as one DFR. Meanwhile, the rule also identifies these steps' product sites to build the DFR's product sites. Next, the extensions and the trigger of the step are transformed into the "requires” DFR constraints.

The middle-level loop is executed for each dimension of the steps. Its rule builds the variation points from the dimensions according to their same types. Its pattern references the "\#dfrFORst" mapping association built by the previous statement. The loop head "dim:Dimension" is combined with building actions. Thus, all the dimensions, having the same type and belonging to a set of steps with the same names, will be merged as one variation point of the DFR that is generated by the set of steps.

The inner loop is executed for each sub-variation of some dimension of some step. Its rule builds a variant through merging a set of sub-variations with the same name.

Note that the CV ratios of DFRs will be calculated according to the product sites of DFRs after the model transformation process. For those steps without subvariations, only the outer loop will be executed for analyzing their commonality and variability.

\section{Related Work and Discussion}

Some researchers have studied how to extend use cases with variability. They exploit and extend the use cases for product lines in different perspectives and by different means. For example, Jacobson et al. [8] introduce variation points into use case diagrams and use them to describe different ways of performing actions within a use case. Gomaa [22] and John and Muthig [13] introduce stereotypes, such as $\langle<$ variant $>>$, $<<$ kernel $>>$ and $<<$ optional $>>$ for use cases for modeling families of systems. Most research remains the documentation of variabilities, but ignores the principle of SPLE, i.e. proactive reuse. Topics such as how to systematically reuse existing requirements to derive domain requirements lack enough research. In this paper, we extend use cases with a multi-dimensional structure for modeling the variability, and record the product site of each step in use cases. These extensions support systematic reuse of domain knowledge by deriving domain requirements from existing use cases, and analyzing the commonality and variability.

Many domain analysis methods, such as FODA (Fea-
ture-Oriented Domain Analysis) [6], FORM (Feature-Oriented Reuse Method) [7], SCV (Scope, Commonality, and Variability) [10], RSEB [8] and FeatuRSEB [9], identify and document the commonalities and variabilities of related systems in a domain. Nevertheless, these typical domain analysis techniques mostly depend on domain experts' knowledge and experience to manually acquire commonalities and variabilities. In addition, Braganca and Machado [23] and Wang et al. [24] propose automated approaches to transformation between feature models and use cases. Our approach complements the existing domain analysis techniques by providing automated support for developing DFRs from use cases.

Manual effort is yet indispensable in our approach. First, analysts must scope domain requirements and formulate domain terminology. Second, each use case must be specified with the predefined use case metamodel and unified domain terminology, which helps uncover the incompleteness and inconsistency of existing requirements to a certain degree. Although NLP techniques could be incorporated into DFRs development to minimize the manual operation cost [4], their accuracy can not yet be guaranteed sufficiently. Third, a comprehendsive analysis of variability dependencies must be done by analysts in terms of domain context. In summary, domain experts still play an essential role in the DFRs development.

\section{Conclusions}

In this paper, we propose an automated approach to DFRs derivation using the metamodeling and model transformation techniques. The main contribution is to present a model-driven approach to domain requirements analysis. The approach complements the existing domain analysis techniques by reducing the manual operation cost and improving the efficiency in DFRs development, which further enhances the transition process from single-system engineering to SPLE. In addition, the model transformation definition documents the traceability information between DFRs and use cases, which helps manage domain requirements and trace their rationale for decision making in SPLE.

Our future work is twofold. First, we will further improve our current approach, especially exploring how to handle the complex constraint dependency. Second, we will verify our approach through an in-depth experimenttal study.

\section{Acknowledgements}

This work is supported by the National Natural Science Foundation of China (NSFC No. 60773088), the National 
High-tech R \& D Program of China (863 Program No. 2009AA04Z106), and the Key Program of Basic Research of Shanghai Municipal S \& T Commission (No. 08JC1411700).

\section{REFERENCES}

[1] P. Clements and L. Northrop, "Software Product Lines: Practices and Patterns,” Addison Wesley, Boston, 2001.

[2] K. Pohl, G. Bockle and F. van der Linden, "Software Product Line Engineering: Foundations, Principles and Techniques,” Springer Verlag, Heidelberg, 2005.

[3] C. W. Krueger, "Easing the Transition to Software Mass Customization,” Proceedings International Workshop on Product Family Engineering, Bilbao, 2001, pp. 282-293.

[4] N. Niu and S. Easterbrook, "Extracting and Modeling Product Line Functional Requirements," Proceedings RE'08, Barcelona, 2008, pp. 155-164.

[5] R. Prieto-Diaz, "Domain Analysis for Reusability," Proceedings 11th Annual International Computer Software and Applications Conference, Tokyo, 1987, pp. 23-29.

[6] K. C. Kang, S. G. Cohen, J. A. Hess, W. E. Novak and A. S. Peterson, "Feature-Oriented Domain Analysis (FODA) Feasibility Study,” Technical Report CMU/SEI-90-TR021, Software Engineering Institute, Canadian Mennonite University, 1990.

[7] K. C. Kang, S. Kim, J. Lee, K. Kim, E. Shin and M. Huh, "FORM: A Feature-Oriented Reuse Method with Domain-Specific Reference Architectures," Annals of Software Engineering, Vol. 5, No. 1, 1998, pp. 143-168.

[8] I. Jacobson, M. Griss and P. Jonsson, "Software Reuse: Architecture Process and Organization for Business Success,” Association for Computing Machinery Press, 1997.

[9] M. L. Griss, J. Favaro and M. D. Alessandro, "Integrating Feature Modeling with the RSEB,” Proceedings of International Conference on Steel Rolling'98, Victoria, 1998, pp. 76-85.

[10] J. Coplien, D. Hoffman and D. Weiss, "Commonality and Variability in Software Engineering," IEEE Software, Vol. 15, No. 6, 1998, pp. 37-45.

[11] M. Moon, H. S. Chae and K. Yeom, “An Approach to Developing Domain Requirements as a Core Asset Based on Commonality and Variability Analysis in a Product Line,” IEEE Transactions on Software Engineering, Vol. 31, No. 7, 2005, pp. 551-569.
[12] E. de Almeida, J. Mascena, A. Cavalcanti, A. Alvaro, V. Garcia, S. de Lemos Meira and D. Lucrédio, "The Domain Analysis Concept Revisited: A Practical Approach," Proceedings of International Conference on Steel Rolling'06, Turin, 2006, pp. 43-57.

[13] I. John and D. Muthig, “Tailoring Use Cases for Product Line Modeling,” Proceedings of Requirements Engineering for Product Lines'02, Essen, 2002, pp. 26-32.

[14] Z. Zhang, "Model Component Reuse-Conceptual Foundations and Application in the Metamodel-Based Systems Analysis and Design Environment,” Ph.D. dissertation, Jyvaskyla Studies in Computing 39, University of Jyvaskyla, 2004.

[15] A. G. Kleppe, J. B. Warmer and W. Bast, "MDA Explained: The Model Driven Architecture: Practice and Promise,” Addison Wesley, Longman Publishing Co., Inc., Boston, 2003.

[16] S. Sendall and W. Kozaczynski, "Model Transformation: The Heart and Soul of Model-Driven Software Development,” IEEE Software, Vol. 20, No. 5, 2003, pp. 42-45.

[17] A. Cockburn, "Basic Use Case Template," 2010. http:// alistair.cockburn.us/Basic+use+case+template.

[18] A. Kalnins and J. B. E. Celms, "Model Transformation Language MOLA," Proceedings of Working Conference on Model Driven Architecture: Foundations and Applications (MDAFA'04), Linköping, 2004, pp. 62-76.

[19] I. Jacobson, "Object-Oriented Software Engineering: A Use Case Driven Approach,” Addison Wesley, Wokingham, England, 1992.

[20] Object Management Group, "Unified Modeling Language (UML), version 2.2,” 2010. http://www.omg.org/technology/documents/formal/uml.htm.

[21] A. Cockburn, "Writing Effective Use Cases,” Addison Wesley, Boston, 2001.

[22] H. Gomaa, "Object Oriented Analysis and Modeling for Families of Systems with UML," Proceedings of International Conference on Steel Rolling'00, Vienna, 2000, pp. 89-99.

[23] A. Braganca and R. J. Machado, “Automating Mappings between Use Case Diagrams and Feature Models for Software Product Lines,” Proceedings of Southern Poverty Law Center'07, Kyoto, 2007, pp. 3-12.

[24] B. Wang, W. Zhang, H. Zhao, Z. Jin and H. Mei, "A Use Case Based Approach to Feature Models' Construction,” Proceedings RE'09, Atlanta, Georgia, 2009, pp. 121-130. 